\title{
Effects of Navier slip and suction/injection on an unsteady reactive variable viscosity non-Newtonian fluid flow in a channel filled with porous medium and convective boundary conditions
}

\author{
L. Rundora ${ }^{1} \&$ O. D. Makinde ${ }^{2}$ \\ ${ }^{1}$ Department of Mathematics, \\ Vaal University of Technology, South Africa \\ ${ }^{2}$ Faculty of Military Science, Stellenbosch University, South Africa
}

\begin{abstract}
This investigation deals with the combined effects of Navier slip, suction/injection, convective cooling and temperature dependent viscosity on an unsteady third grade fluid flow in a porous channel filled with saturated porous medium. The heat exchange with the ambient at the channel surfaces is assumed to obey Newton's law of cooling. The model equations governing the flow system are solved using a semi-implicit finite difference scheme. The effects of the thermophysical parameters on the temperature and velocity fields, the skin friction and the wall heat transfer rate are simulated and discussed in detail.

Keywords: third grade fluid, Navier slip, suction/injection, temperature dependent viscosity, convective boundary conditions.
\end{abstract}

\section{Introduction}

Theoretical consideration of pressurized unsteady fluid flow involving heat and mass transfer in porous media is a subject that has aroused a lot of research interest within the scientific community in the modern era. Such interest is owing to the wide industrial applications [1]. Since such flows are associated with inherent exothermic chemical reactions, safety concerns are also a major issue that warrants unending investigations. Fluid flow in channels with porous walls 
has been investigated by, among other researchers, Beg and Makinde [2], Chinyoka and Makinde [3, 4], Makinde and Ogulu [5], Wang et al. [6], and Attia [7]. Regenerative heat exchange and adsorption, surface catalysis of chemical reactions, oil and gas production, insulation of buildings, heat and fluid exchange inside human and animal organs, drying of food, nuclear waste disposal, and geothermal energy extraction are examples of applicable areas where thermal stability criteria is an important aspect of the scientific analyses.

More recent focus has been on non-Newtonian fluid flows. Non-Newtonian is a generic term that characterizes fluids exhibiting non-linear viscoelastic behaviour that cannot be modelled by the classical Navier-Stokes equations [8]. Examples of such reactive fluids are polymeric liquids, suspensions, liquid crystals, liquid metals and alloys, nuclear fuel slurries, paints, heavy oils and grease. Amongst the models that have been postulated to describe such rheological characteristics are the fluids of the differential type exemplified by third grade fluids $[9,10]$. Introducing uniform suction/injection at the channel walls is a means of mitigating the thermal effects of highly exothermic, reactive viscoelastic fluids [11]. The combined effects of unsteadiness, suction/injection, temperature dependent viscosity and asymmetric convective boundary conditions on the thermal stability condition for a reactive third grade Poiseuille flow was reported in Rundora and Makinde [12]. In the current installment we investigate the combined effects of suction/injection and Navier slip at the channel walls on the heat transfer characteristics in such flows. Evidences of slip of a fluid on a solid surface were reported by several authors [13, 14]. Meanwhile studies involving flow and heat transfer in channels with wall slip are important as they can lead to the improvement of design and operation of many industrial and engineering devices.

The remainder of this paper is organized as follows: in section 2 we establish the mathematical formulation of the problem, in section 3 a semi-implicit finite difference scheme is utilized to numerically solve the problem, and numerical and graphical results are displayed and discussed quantitatively with respect to the various thermophysical parameters embedded in the system in section 4 .

\section{Mathematical model formulation}

An unsteady flow of an incompressible temperature dependent viscosity, reactive third grade fluid through a channel filled with a homogeneous and isotropic porous medium is examined. The channel walls are assumed to be uniformly porous and that fluid injection is through the lower wall while suction occurs at the upper wall. The plate surfaces are also subjected to asymmetric convective heat exchange with the surrounding medium as a result of unequal heat transfer coefficients and the fluid motion is as a result of an applied axial pressure gradient. Fig. 1 depicts the schematics of the problem.

Following Brinkman [1], Truesdell and Noll [8], Rajagopal [9], Fosdick and Rajagopal [10], Som et al. [15], and neglecting the reacting viscous fluid consumption, the governing equations for the momentum and heat balance can be written as 


$$
\begin{aligned}
& \rho\left(\frac{\partial u}{\partial \bar{t}}+V \frac{\partial u}{\partial \bar{y}}\right)=\frac{\partial \bar{P}}{\partial \bar{x}}+\frac{\partial}{\partial \bar{y}}\left[\bar{\mu}(T) \frac{\partial u}{\partial \bar{y}}\right]+\alpha_{1} \frac{\partial^{3} u}{\partial \bar{y}^{2} \partial \bar{t}}+6 \beta_{3} \frac{\partial^{2} u}{\partial \bar{y}^{2}}\left(\frac{\partial u}{\partial \bar{y}}\right)^{2}-\frac{\bar{\mu}(T) u}{\rho K} \\
& \rho c_{p}\left(\frac{\partial T}{\partial \bar{t}}+V \frac{\partial T}{\partial \bar{y}}\right)=k \frac{\partial^{2} T}{\partial \bar{y}^{2}}+\left(\frac{\partial u}{\partial \bar{y}}\right)^{2}\left(\bar{\mu}(T)+2 \beta_{3}\left(\frac{\partial u}{\partial \bar{y}}\right)^{2}\right)+\frac{\bar{\mu}(T) u^{2}}{K}+Q C_{0} A\left(\frac{h T}{v}\right)^{m} e^{-\frac{E}{R T}}
\end{aligned}
$$

The additional viscous dissipation term in equation (2) is valid in the limit of very small and very large porous medium permeability. The appropriate initial and boundary conditions are

$$
\begin{gathered}
u(\bar{y}, 0)=0, \quad T(\bar{y}, 0)=T_{0}, \\
\beta_{0} u(0, \bar{t})=\bar{\mu}(T) \frac{\partial u}{\partial \bar{y}}(0, \bar{t}),-k \frac{\partial T}{\partial \bar{y}}(0, \bar{t})=h_{1}\left[T_{a}-T(0, \bar{t})\right], \\
\beta_{1} u(a, \bar{t})=\bar{\mu}(T) \frac{\partial u}{\partial \bar{y}}(a, \bar{t}),-k \frac{\partial T}{\partial \bar{y}}(a, \bar{t})=h_{2}\left[T(a, \bar{t})-T_{a}\right] .
\end{gathered}
$$

Here $T$ is the absolute temperature, $\rho$ is the density, $c_{p}$ is the specific heat at constant pressure, $\bar{t}$ is the time, $h_{1}$ is the heat transfer coefficient at the lower plate, $h_{2}$ is the heat transfer coefficient at the upper plate, $T_{0}$ is the fluid initial temperature, $T_{a}$ is the ambient temperature, $k$ is the thermal conductivity of the material, $Q$ is the heat of reaction, $A$ is the rate constant, $E$ is the activation energy, $R$ is the universal gas constant, $C_{0}$ is the initial concentration of the reactant species, $a$ is the channel width, $l$ is Planck's number, $h$ is Boltzmann's constant, $v$ is the vibration frequency, $K$ is the porous medium permeability, $\alpha_{1}$ and $\beta_{3}$ are the material coefficients, $\bar{P}$ is the modified pressure, and $m$ is the numerical exponent such that $m \in\{-2,0,0.5\}$, where the three values represent numerical exponents for sensitized, Arrhenius and

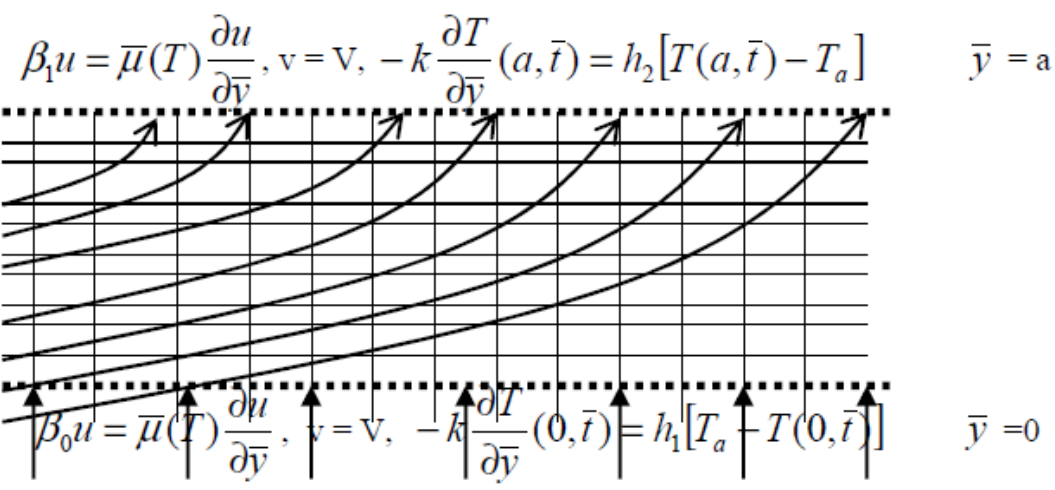

Figure 1: Problem schematics and coordinate system. 
bimolecular kinetics respectively (see Makinde [16, 17] and Frank-Kamenetskii [18]). The temperature dependent viscosity $(\bar{\mu})$ can be expressed as

$$
\bar{\mu}(T)=\mu_{0} e^{-b\left(T-T_{0}\right)},
$$

where $b$ is a viscosity variation parameter and $\mu_{0}$ is the initial fluid dynamic viscosity at temperature $T_{0}$. We introduce the following dimensionless variables into eqns. (1)-(6);

$$
\begin{aligned}
& y=\frac{\bar{y}}{a}, \alpha=\frac{b R T_{0}^{2}}{E}, w=\frac{u \rho a}{\mu_{0}}, \theta=\frac{E\left(T-T_{0}\right)}{R T_{0}^{2}}, \theta_{a}=\frac{E\left(T_{a}-T_{0}\right)}{R T_{0}^{2}}, \operatorname{Re}=\frac{V a \rho}{\mu_{0}}, \gamma=\frac{\beta_{3} \mu_{0}}{\rho^{2} a^{4}}, \delta=\frac{\alpha_{1}}{\rho a^{2}}, \\
& B i_{1}=\frac{h_{1} a}{k}, B i_{2}=\frac{h_{2} a}{k}, D a=\frac{K}{a^{2}}, \operatorname{Pr}=\frac{\mu_{0} c_{p}}{k}, \varepsilon=\frac{R T_{0}}{E}, x=\frac{\bar{x}}{a}, P=\frac{\bar{P} \rho a^{2}}{\mu_{0}^{2}}, G=-\frac{\partial \bar{P}}{\partial \bar{x}}, S^{2}=\frac{1}{D a}, \\
& t=\frac{\bar{t} \mu_{0}}{\rho a^{2}}, n_{1}=\frac{\mu_{0}}{\beta_{0} a}, \mu=\frac{\bar{\mu}}{\mu_{0}}, \lambda=\left(\frac{h T_{0}}{v}\right)^{m} \frac{Q E A a^{2} C_{0} e^{-\frac{E}{R T}}}{T_{0}^{2} R k}, \Omega=\left(\frac{v}{h T_{0}}\right)^{m} \frac{\mu_{0}^{3} e^{\frac{E}{R T}}}{\rho^{2} Q A a^{4} C_{0}}, n_{2}=\frac{\mu_{0}}{\beta_{1} a}
\end{aligned}
$$

and obtain the following dimensionless governing equations:

$$
\begin{gathered}
\frac{\partial w}{\partial t}+\operatorname{Re} \frac{\partial w}{\partial y}=G-S^{2} w e^{-\alpha \theta}+e^{-\alpha \theta} \frac{\partial^{2} w}{\partial y^{2}}-\alpha e^{-\alpha \theta} \frac{\partial \theta}{\partial y} \frac{\partial w}{\partial y}+\delta \frac{\partial^{3} w}{\partial y^{2} \partial t}+6 \gamma \frac{\partial^{2} w}{\partial y^{2}}\left(\frac{\partial w}{\partial y}\right)^{2}, \\
\operatorname{Pr} \frac{\partial \theta}{\partial t}+\operatorname{Pr} \operatorname{Re} \frac{\partial \theta}{\partial y}=\frac{\partial^{2} \theta}{\partial y^{2}}+ \\
\lambda\left\{(1+\varepsilon \theta)^{m} \exp \left(\frac{\theta}{1+\varepsilon \theta}\right)+\Omega\left[S^{2} w^{2} e^{-\alpha \theta}+\left(\frac{\partial w}{\partial y}\right)^{2}\left(e^{-\alpha \theta}+2 \gamma\left(\frac{\partial w}{\partial y}\right)^{2}\right)\right]\right\} \\
w(y, 0)=0, \quad \theta(y, 0)=0, \\
w(0, t)=n_{1} e^{-\alpha \theta(0, t)} \frac{\partial w}{\partial y}(0, t), \quad \frac{\partial \theta}{\partial y}(0, t)=-B i_{1}\left[\theta_{a}-\theta(0, t)\right], \\
w(1, t)=n_{2} e^{-\alpha \theta(1, t)} \frac{\partial w}{\partial y}(1, t), \quad \frac{\partial \theta}{\partial y}(1, t)=-B i_{2}\left[\theta(1, t)-\theta_{a}\right],
\end{gathered}
$$

where $\lambda$ represents the Frank-Kamenetskii parameter, $n_{1}, n_{2}$ are the lower wall slip parameter and the upper wall slip parameter respectively, $\operatorname{Pr}$ is the Prandtl number, $B i$ is the Biot number, $\varepsilon$ is the activation energy parameter, $\delta$ is the material parameter, $\gamma$ is the non-Newtonian parameter, $G$ is the pressure gradient parameter, $D a$ is the Darcy number, $\alpha$ is the variable viscosity parameter, $\Omega$ is the viscous heating parameter, $\theta_{a}$ is the ambient temperature parameter, $S$ is the porous medium shape parameter, and $\mathrm{Re}$ is the suction /injection Reynolds number.

The skin friction $\left(C_{f}\right)$ at the channel walls is given as

$$
C_{f}=\frac{\rho \alpha^{2} \tau_{w}}{\mu_{0}^{2}}=\left.e^{-\alpha \theta} \frac{d w}{d y}\right|_{y=0,1}
$$


where $\tau_{w}=\bar{\mu}(T) \frac{\partial u}{\partial \bar{y}}$ is the shear stress evaluated at the wall $\bar{y}=0, a$. The other dimensionless quantity of interest is the wall heat transfer rate $(N u)$ given by

$$
N u=-\frac{d \theta}{d y}(1, t)
$$

In the following section, eqns. (8)-(14) are solved numerically using a semiimplicit finite difference scheme.

\section{Numerical solution}

The numerical algorithm is based on the semi-discretization finite-difference scheme in Chinyoka [11, 19]. The discretization of the governing equations is based on a linear Cartesian mesh and uniform grid on which finite differences are taken. Both the second and first spatial derivatives are approximated by second-order central differences. The equations corresponding to the first and last grid points are modified to incorporate the boundary conditions. The resulting nonlinear system of the initial value problem is then solved iteratively using the initial value solvers like the shooting method or the Runge-Kutta Fehlberg integration scheme [20]. The semi-discretization scheme for the velocity and temperature component read as:

$$
\begin{aligned}
& \frac{d}{d t}\left[w_{i}-\delta\left(\frac{w_{i+1}-2 w_{i}+w_{i-1}}{(\Delta y)^{2}}\right)\right]=G-S^{2} e^{-\alpha \theta_{i}} w_{i}-\operatorname{Re}\left(\frac{w_{i+1}-w_{i-1}}{2 \Delta y}\right) \\
& +\frac{\left(w_{i+1}-2 w_{i}+w_{i-1}\right)}{(\Delta y)^{2}}\left[e^{-\alpha \theta_{i}}+6 \gamma\left(\frac{w_{i+1}-w_{i-1}}{2 \Delta y}\right)^{2}\right]-\alpha e^{-\alpha \theta_{i}}\left(\frac{w_{i+1}-w_{i-1}}{2 \Delta y}\right)\left(\frac{\theta_{i+1}-\theta_{i-1}}{2 \Delta y}\right) \\
& \operatorname{Pr} \frac{d \theta_{i}}{d t}=\frac{\left(\theta_{i+1}-2 \theta_{i}+\theta_{i-1}\right)}{(\Delta y)^{2}}-\operatorname{Pr} \operatorname{Re} \frac{\left(\theta_{i+1}-\theta_{i-1}\right)}{2 \Delta y}+ \\
& \lambda\left\{\left[\left(1+\varepsilon \theta_{i}\right)^{m} \exp \left(\frac{\theta_{i}}{1+\varepsilon \theta_{i}}\right)\right]+\Omega\left[S^{2} w_{i}^{2} e^{-\alpha \theta_{i}}+\left(\frac{w_{i+1}-w_{i-1}}{2 \Delta y}\right)^{2}\left(e^{-\alpha \theta_{i}}+2 \gamma\left(\frac{w_{i+1}-w_{i-1}}{2 \Delta y}\right)^{2}\right)\right]\right\}
\end{aligned}
$$

with initial conditions

$$
w_{i}(0)=0, \theta_{i}(0)=0
$$

where $w_{i}(t)=w\left(y_{i}, t\right), \theta_{i}(t)=\theta\left(y_{i}, t\right)$ and the spatial interval $[0,1]$ is partitioned into $N$ equal subintervals. The grid size and the grid points are defined as $\Delta y=\frac{1}{N}$ and $y_{i}=(i-1) \Delta y, 1 \leq i \leq N+1$.

\section{Results and discussion}

\subsection{Transient and steady state profiles}

Fig. 2 displays transient and steady state velocity and temperature profiles in the absence of fluid injection/suction. In both cases we have parabolic profiles with a 


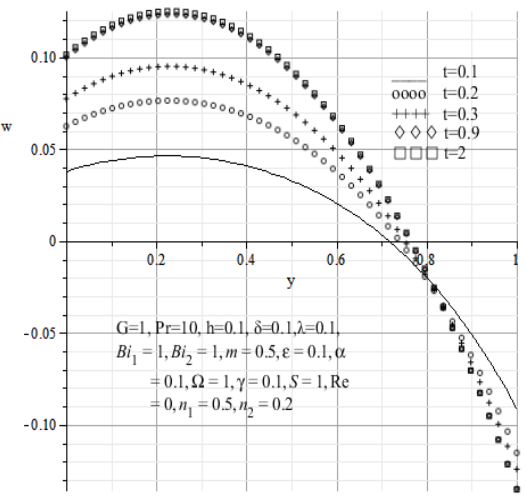

(a)

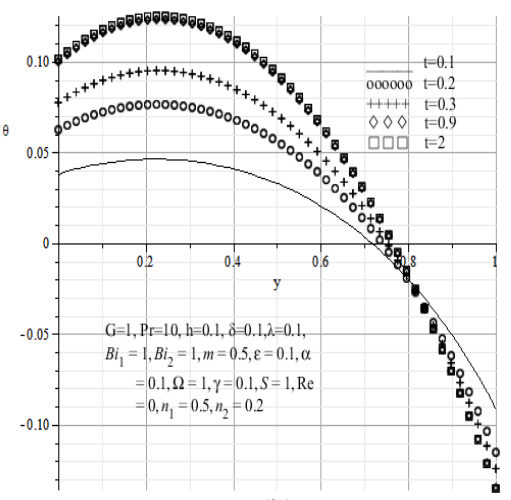

(b)

Figure 2: (a) Velocity and (b) temperature profiles with no suction/injection.

transient increase until steady state is reached. Negative velocity profiles indicate fluid flow reversal due to velocity slip at the channel walls.

\subsection{Parameter dependency of solutions}

In this investigation fluid is considered to be injected uniformly through the lower wall and suction occurs out of the upper wall. As a result of this, the velocity and temperature profiles will tend to be shifted towards the upper wall.

Fig. 3 shows velocity and temperature profiles diminishing with an increase in the suction injection Reynolds number. As fluid is sucked out of the upper wall, the velocity towards the upper wall is higher than elsewhere in the channel and this in turn increases the rate of convective heat transfer out of the upper wall resulting in a significant drop in fluid temperature. An increase in the porous medium shape parameter will make it difficult for the fluid particles to enter the reduced pore spaces, and this significantly retards the fluid velocity.
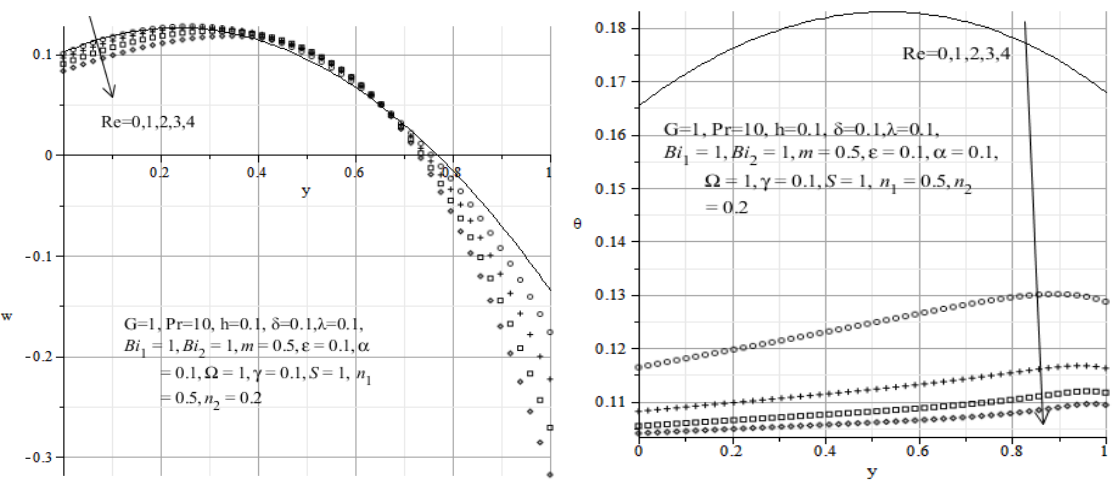

Figure 3: Velocity and temperature profiles with increasing Re. 

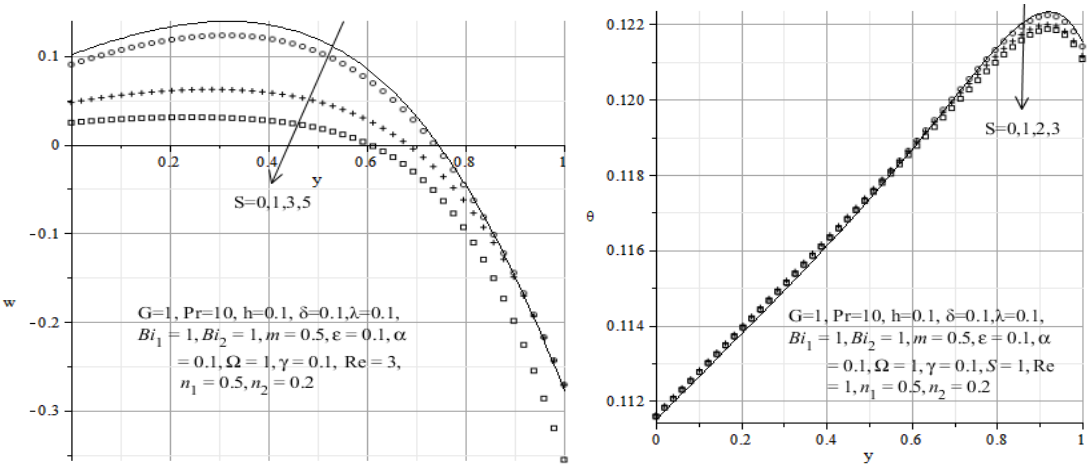

Figure 4: $\quad$ Velocity and temperature profiles with increasing $S$.

The fluid temperature is in turn decreased too as a result of a significant decrease in the strength of the viscous heating source terms in the temperature equation.

Fig. 4 captures this phenomenon. Fig. 5 pictures a significant rise in fluid velocity with an increase in the temperature dependent viscosity. The corresponding effect of variable viscosity on fluid temperature is marginal.

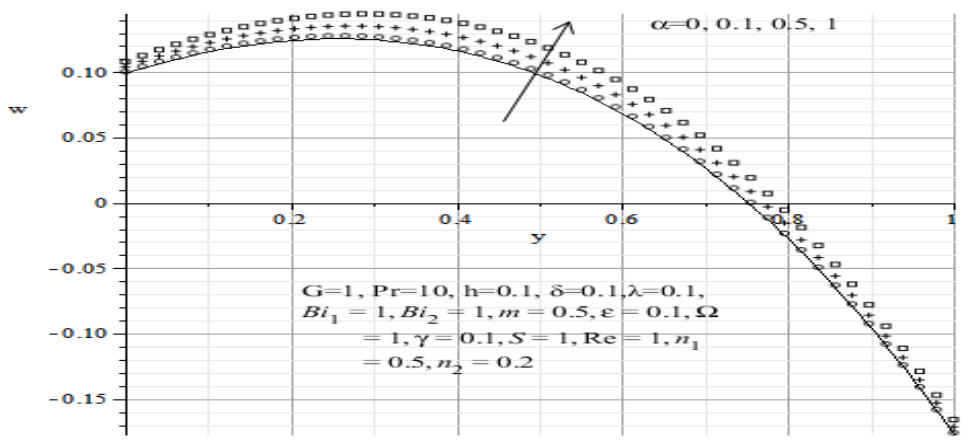

Figure 5: $\quad$ Velocity profiles with increasing $\alpha$.

The Frank-Kamenetskii parameter, $\lambda$, whose effect is to increase the rate of the exothermic chemical reaction, is observed to significantly increase the fluid temperature (see fig. 6). The effect on fluid velocity is unnoticeable. Figs 7 and 8 show the effect of the wall slip parameters on fluid velocity and temperature profiles. Fluid velocity is observed to increase significantly with increasing magnitude of the lower wall slip parameter, $n_{1}$, whereas an increase in the upper wall slip parameter, $n_{2}$, significantly diminishes fluid velocity. A possible explanation of this phenomenon is that flow reversal at the upper wall will inevitably reduce fluid velocity. This flow reversal causes rapid mixing of fluid particles and results in friction that induces a raise in the fluid temperature. 


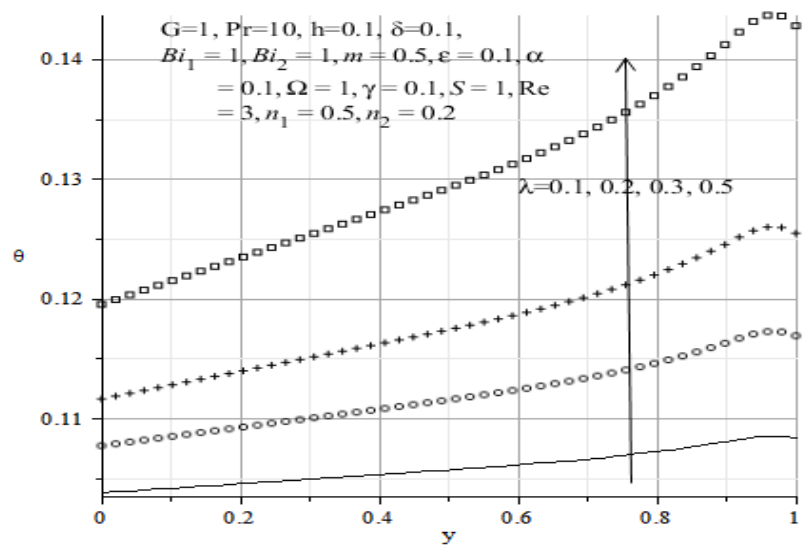

Figure 6: Temperature profiles with increasing $\lambda$.
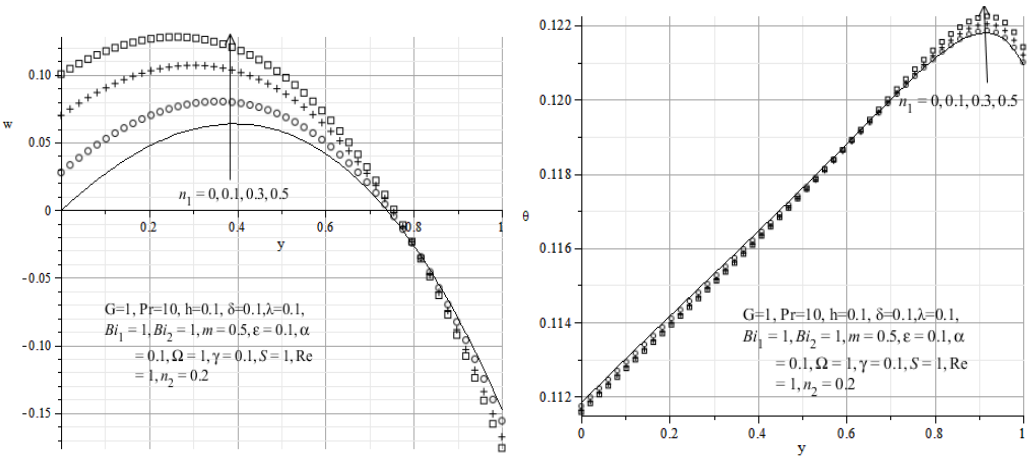

Figure 7: Velocity and temperature profiles with increasing $n_{1}$.
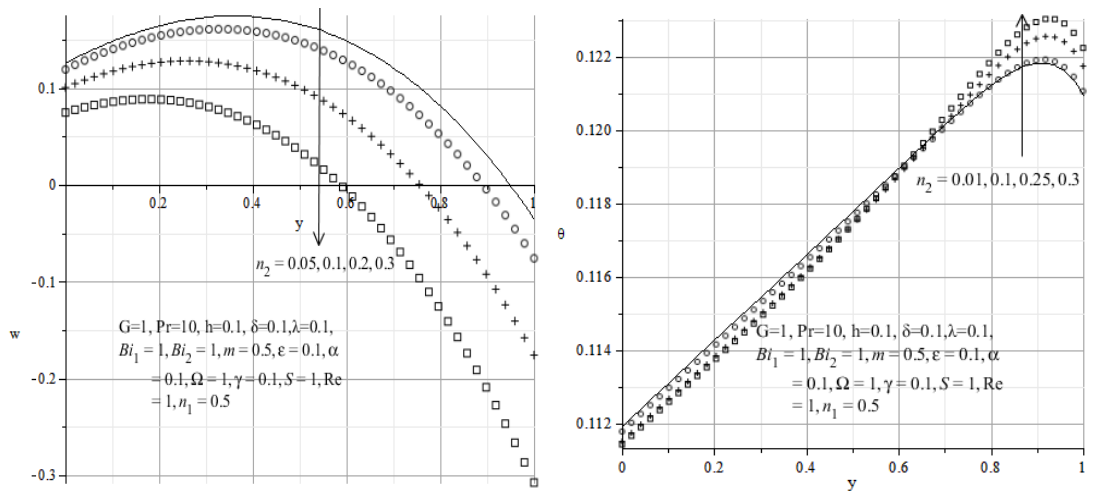

Figure 8: Velocity and temperature profiles with increasing $n_{2}$. 


\subsection{Skin friction and Nusselt number}

Fig. 9 depicts skin friction profiles. Increasing the suction/injection Reynolds number, the Prandtl number and the upper wall slip parameter increases the skin friction tremendously. On the other hand the variable viscosity parameter is observed to diminish the skin friction significantly.

Fig. 10 depicts the rate of heat transfer at the channel walls (Nusselt number). The wall heat transfer rate is seen to diminish with increasing magnitude of the suction/injection Reynolds number and the Prandtl number. It increases with the increase in the upper wall slip parameter.
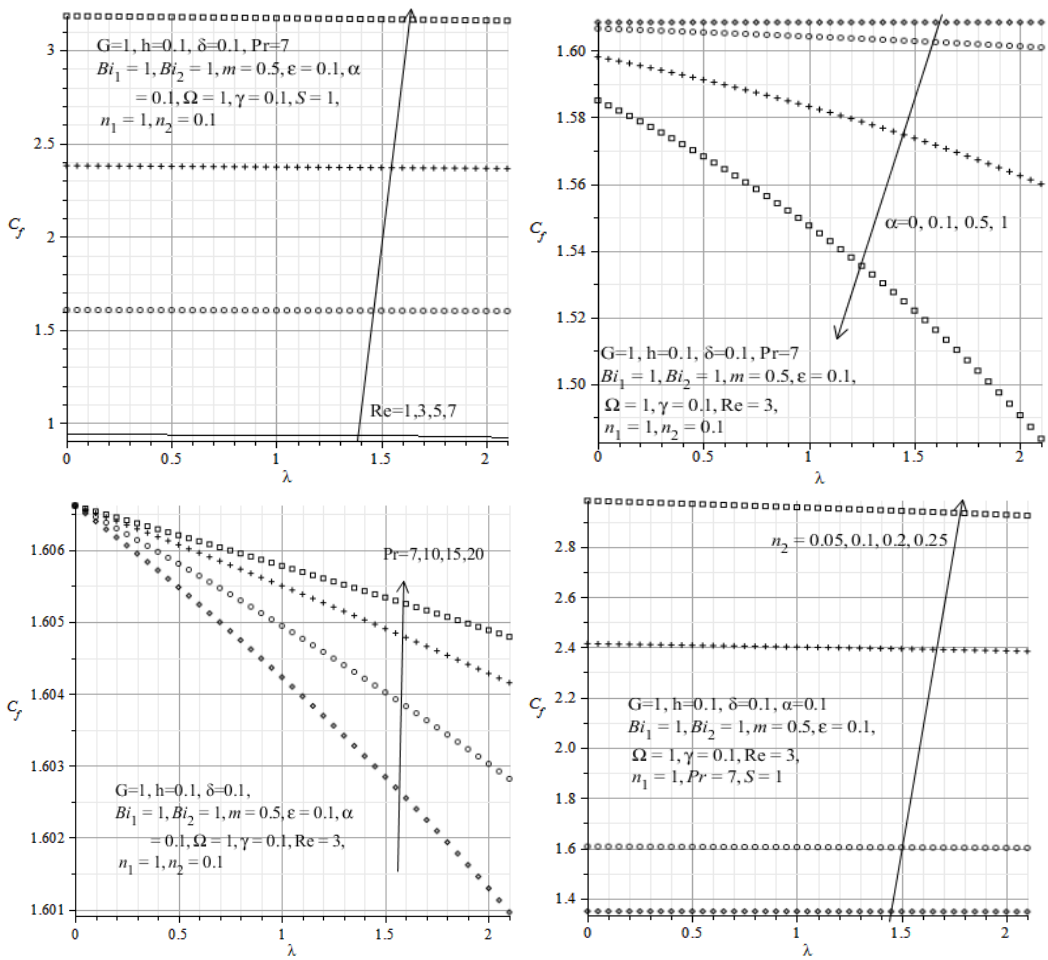

Figure 9: Skin friction with increasing $\operatorname{Re}$ and $\lambda, \alpha$ and $\lambda, \operatorname{Pr}$ and $\lambda, n_{2}$ and $\lambda$.

\subsection{Blow up of solutions}

A significant aspect of this study is to determine the thermophysical parameter values that can combat runaway fluid temperatures that may lead to chemical explosions. One such parameter that requires careful monitoring and control is the reaction parameter, $\lambda$. Fig. 11 shows that at low suction/injection Reynolds numbers and Prandtl numbers, the temperature blow up value of the reaction 
parameter is very low. On the contrary, the temperature blow up value of $\lambda$ is higher at high suction/injection Reynolds numbers and high Prandtl numbers. It is thus significantly safer to run the process at higher suction/injection Reynolds numbers and higher Prandtl numbers.

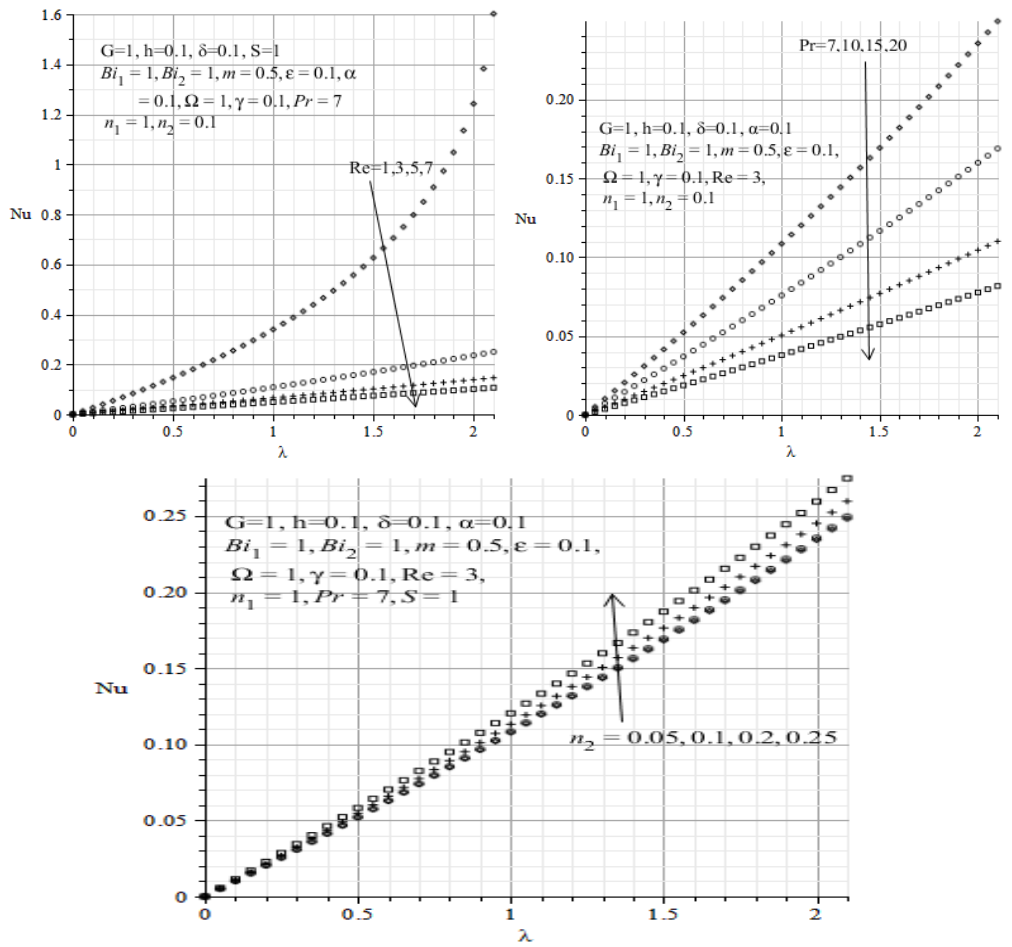

Figure 10: Nusselt number with increasing $\operatorname{Re}$ and $\lambda, \operatorname{Pr}$ and $\lambda, n_{2}$ and $\lambda$.

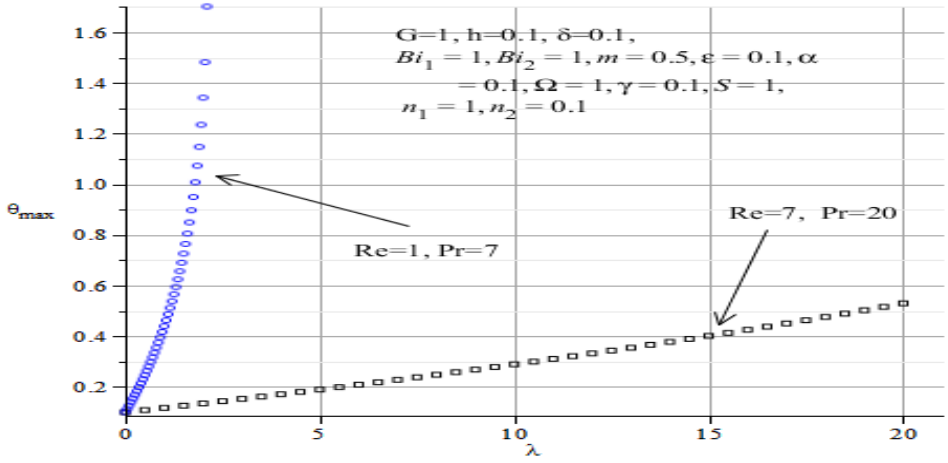

Figure 11: Blow up of solution. 


\section{Conclusion}

The combined effects of Navier slip and suction/injection on unsteady reactive variable viscosity non-Newtonian fluid flow in a channel filled with porous medium and convective boundary conditions are investigated. We point out some of the important features of physical interest arising from the study.

The velocity and temperature fields are retarded by the suction injection Reynolds number. The lower wall slip parameter increases the velocity field, whereas the upper wall slip parameter retards it. Fluid temperature is increased by both the lower wall and upper wall slip parameters. Skin friction is increased by the suction/injection Reynolds number, the Prandtl number and the upper wall slip parameter. The porous medium shape parameter and the variable viscosity parameter have the opposite effect. The wall heat transfer rate is diminished by the suction/injection Reynolds number and the Prandtl number, whereas the upper wall slip parameter increases it. At higher suction/injection Reynolds numbers and Prandtl numbers, blow up of solution is delayed significantly.

\section{References}

[1] Brinkman, H.C., On the permeability of media consisting of closely packed porous particles, Appl. Sci. Res. A1, pp. 81-86, 1947.

[2] Beg, O.A. \& Makinde, O.D., Viscoelastic flow and species transfer in a Darcian high-permeability channel, J. Pet. Sci. Eng. 76, pp. 93-99, 2011.

[3] Chinyoka, T. \& Makinde, O.D., Computational dynamics of unsteady flow of a variable viscosity reactive fluid in a porous pipe, Mech. Res. Commun. 37, pp. 347-353, 2010.

[4] Makinde, O.D. \& Chinyoka, T., Analysis of unsteady flow of a variable viscosity reactive fluid in a slit with wall suction or injection, J. Pet. Sci. Eng., 94-95, pp. 1-11, 2012.

[5] Makinde, O.D. \& Ogulu, A., The Effect of Thermal Radiation on the Heat and Mass Transfer Flow of a Variable Viscosity Fluid Past a Vertical Porous Plate Permeated by a Transverse Magnetic Field, Chem. Eng. Comm., 195, pp. 1575-1584, 2008.

[6] Wang, J., Gao, Z., Gan, G. \& Wu, D., Analytical solution of flow coefficients for a uniformly distributed porous channel, Chem. Eng. Sci. 84 (1), pp. 1-6, 2001.

[7] Attia, H.A., The effect of suction and injection on the unsteady flow between two parallel plates with variable properties, Tamkang J. Sci. Eng. 8(1), pp. 17-22, 2005.

[8] Truesdell, C. \& Noll, W., The non-linear field theories of mechanics, in: Handbuch der Physik (Edited by S. Flugge), Vol. 111/3, Springer, Berlin, 1965.

[9] Rajagopal, K.R., On Boundary Conditions for Fluids of the Differential type: Navier-Stokes Equations and Related Non-linear Problems, Plenum Press, New York, pp. 273, 1995. 
[10] Fosdick, R.L. \& Rajagopal, K.R., Thermodynamics and stability of fluids of third grade, Proc. R. Soc. London A339, pp. 351, 1980.

[11] Chinyoka, T., Suction-injection control of shear banding in non-isothermal and exothermic channel flow of Johnson-Segalman liquids, Trans. ASME J. Fluids Eng. 133 (7), 071205 (12pp.), 2011.

[12] Rundora, L. \& Makinde, O.D., Effects of suction/injection on unsteady reactive variable viscosity non-Newtonian fluid flow in a channel filled with porous medium and convective boundary conditions, J. Pet. Sci. Eng., 108, pp. 328-335, 2013.

[13] Mathews, M.T. \& Hill, J.M., Newtonian flow with nonlinear Navier boundary condition, Acta Mech., vol. 191(3-4), pp. 195, 2007.

[14] Zhu, Y.X. \& Granick, S., Rate-dependent slip of Newtonian liquid at smooth surfaces-art. No. 096105, Phys. Rew, Lett., 8709(9) , pp. 6105, 2001.

[15] Som, S.K., Mondal, S.S. \& Dash, S.K., Energy and energy balance in the process of pulverized coal combustion in a tubular combustor, J. Heat Transfer 127, pp. 1322-1333, 2005.

[16] Makinde, O.D., Thermal ignition in a reactive viscous flow through a channel filled with a porous medium, Trans. ASME J. Heat Transfer. 128, pp. 601-604, 2006.

[17] Makinde, O.D., Thermal stability of a reactive viscous flow through a porous -saturated channel with convective boundary conditions, Appl. Therm. Eng. 29, pp. 1773-1777, 2009.

[18] Frank-Kamenetskii, D.A., Diffusion and Heat Transfer in Chemical Kinetics, Plenum Press, New York, 1969.

[19] Chinyoka, T., Computational dynamics of a thermally decomposable viscoelastic lubricant under shear, Trans. ASME J. Fluids Eng. 130 (12), 121201. (7pp.), 2008.

[20] Na, T.Y., Computational methods in engineering boundary value problems, Academic Press, New York, 1979. 